\title{
Effect of the Diamagnetic term in the ultra-strong coupling regime
}

\author{
Z. Sakhi, A. Chentouf and M. Bennai \\ Physics and Quantum Technology team, Condensed Matter Physics Laboratory, \\ Ben M'sik Faculty of Sciences, Hassan II Casablanca University Morocco \\ E-mail: mdbennai@yahoo.fr
}

\begin{abstract}
We consider a set of two level atoms interacting with a single quantized bosonic mode governed by the Dicke model. In this model it is well known that under a critical value of the light-matter coupling a spontaneous radiation process takes place. In the present work, we investigate the dynamics of the system and we study the Wigner distribution function to visualize the effect of the minimal coupling on the ground state wavefunction from the normal phase to the superradiant one. We show also that the entanglement of Bi-partite model is limited by the presence of the diamagnetic term.
\end{abstract}

Keywords: Entanglement, diamagnetic term, Cavity QED, Circuit QED.

PACS numbers: 03.67.Lx, 85.25.Dq, 42.50.Dv.

\section{Introduction}

The analysis of light-matter interactions described by quantum electrodynamics forms a major subject of quantum optics and quantum information. It has gained a lot of interest in the last few years especially in the context of the Rabi Model [1] and the Dicke Model [2].

This latter, a major paradigm of light-matter interaction coupling, describes an ensemble of $\mathrm{N}$ identical two-level atoms (qubits) coupled to a single quantized mode of a bosonic field. The model was applied in optical cavity QED [3], in the circuit QED [4], and much more recently in a spin-1 Dicke model based on magnetic sublevels of the lowest hyperfine level of 84Rb [5]. Moreover, the advent of quantum information science and the emergence of a novel quantum technologies including quantum superconducting circuits [6] and trapped ions [7], have enabled the exploration of new exotic coupling regimes that were previously inaccessible to experimentation, as the ultra-strong [8] and the deep strong [9] coupling regimes. Those regimes exhibit some interesting physics and can be investigated by the mean of quantum simulations with a high degree of precision and controllability. The ultrastrong coupling regime is a fascinating field of light-matter interaction. It can be reached when the interaction rate is engineered to be faster than the dissipation rate. In this regime, some new dynamics and exotic features that are currently out of reach appear.

As a matter of fact, when an ensemble of atoms interacts with a common radiation field, each atom can no longer be regarded as an individual radiation source but the whole set of atoms can be regarded as a macroscopic dipole moment [10]. A similar phenomenon occurs for a system under the thermodynamic limit near the zero temperature by tuning the light-matter coupling across a quantum critical point. Recall that this phenomenon is known as quantum phase transition(QPT). 
In the thermodynamic limit, Dicke Model presents a superradiant phase transition, for a certain value of the light-matter coupling [11]. In this context, a pioneering work was done by Hepp \& Lieb [12], who have studied the existence of a second order phase transition using the partition function method. On the other hand, Nataf et al [13] have also demonstrated the presence of a no-go theorem that forbids the existence of the superradiant quantum phase transitions in cavity QED. Finally, Baden et al [14] realized an open version of the Dicke model by coupling two hyperfine ground states using two Cavity-assisted Raman transitions.

The fundamental description of the interaction of atomic matter with bosonic fields in the CoulombGauge is known to present terms like the A square vector potential. This latter behaves Diamagnetically, takes its origin from the minimal coupling (Gauge-Coulomb invariance), and stems from the kinetic part of the hamiltonian. It is known that the introduction of the D term in systems hamiltonian provide a rich discussion starring the occurrence of the superradiant phase transition (SPT). Being the subject of several critics $[12,13,15]$, it has led to an interesting point of view assuming the presence of the no-go theorem in the cavity QED. The impact of the diamagnetic on the properties of the ground state wave function, the dynamics of the sub-systems, and more importantly, on the entanglement phenomenon, are not yet well studied.

The specific form of the effective Dicke hamiltonian represents a very discussed topic in the literature, especially concerning the presence of a diamagnetic term D. This term originates from the Coulomb gauge minimal coupling hamiltonian and describes the interaction of a quantized bosonic mode with a non-relativistic charged particles. In the context of circuit QED, Nataf [16] has shown that this term can be avoided by appropriately tuning the Circuit parameters. This discovery initiated a great deal of debate [15]. On the other hand, in the cavity QED, the presence of a such term was argued by several authors $[13,17,18]$, and it's importance is well known in limiting the effectively achievable light-matter coupling [19] and in preventing the superradiant phase transition [20].

In the second section of the present work, we analyze the effect of the D-term on the energy spectrum for the Dicke model. In the third section, we study the phase-space properties of the Dicke model by using the Wigner distribution function to visualize the effect of the minimal coupling on the ground state wavefunction in the normal phase and in the superradiant one. In the fourth section, the entanglement of this bi-partite model is studied and we show that it is limited by the presence of the diamagnetic term. The last section is devoted to discussion and conclusion.

\section{Dicke Model}

The quantum Dicke model is a major paradigm for light-matter interaction [21]. It represents a promising candidate to study the many body problems. This model can describe both the circuit QED and the cavity QED, with $\mathrm{N}$ identical (artificial) atoms. The system is considered in the ultra-strong coupling regime that promises an exciting physics. In this regime, the rotating-wave approximation (RWA) becomes non relevant. This is due to the large values of light-matter coupling, where the counter-rotating terms play a crucial role and herefore can not be neglected.

To well explain the influence of the diamagnetic term on the presence of the second order phase transition, it is convenient to study the excitation spectrum. We consider the Dicke hamiltonian, a quantum-optical model describing the interaction of $\mathrm{N}$ two-level atoms (artificial) atoms (qubits) coupled to a single quantized bosonic mode. The system hamiltonian is given by: 


$$
H=w_{0} \sum s_{Z}+w a^{+} a+D\left(a+a^{+}\right)^{2}+\sum \frac{\lambda}{\sqrt{N}}\left(a+a^{+}\right)\left(s^{-}+s^{+}\right)
$$

where, $w_{0}$ denotes the energy level splitting between the two level systems, a+, a are the creation and annihilation operators of the bosonic field. The spin-half Pauli matrices $\left(S_{Z}, S^{-}, S^{+}\right)$obey the commutation rules $\left[s^{+}, s^{-}\right]=2 s_{z}$ and $\left[s_{z}, s^{ \pm}\right]= \pm s^{ \pm}, \lambda$ is the light-matter coupling for $\mathrm{N}$ identical qubits. The D term represents the Gauge minimal coupling of atoms which will play a crucial role in our study.

The Dicke hamiltonian is further simplified by using both, $j$ the length of the pseudo-spin $(j=N / 2)$ and the collective atomic operators : $J_{z}=\sum s_{z} ; J^{ \pm}=\sum s^{ \pm}$so the hamiltonian is:

$$
H=w_{0} J_{Z}+w a^{+} a+D\left(a+a^{+}\right)^{2}+\frac{\lambda}{\sqrt{2 j}}\left(a+a^{+}\right)\left(J_{-}+J_{+}\right)
$$

where, $J_{z}, \mathrm{~J} \pm$ obey the usual angular momentum commutation relations.

In order to study the excitation spectrum in the thermodynamic limit, one must resort to the Holstein-Primakoff transformation of the angular momentum operators, given by :

$$
J_{+}=b^{+} \sqrt{2 j-b^{+} b}, J_{-}=b \sqrt{2 j-b^{+} b} \text { and } J_{z}=b^{+} b-j
$$

$\mathrm{b}^{+}$and $\mathrm{b}$ are the bosonic creation and annihilation operators such that $\left[\mathrm{b}^{+}, \mathrm{b}\right]=1$. The hamiltonian takes the form

$$
H=w_{0}\left(b^{+} b-j\right)+w a^{+} a+D\left(a+a^{+}\right)^{2}+\lambda\left(a+a^{+}\right)\left(b^{+} \sqrt{1-\frac{b^{+} b}{2 j}}+b \sqrt{1-\frac{b^{+} b}{2 j}}\right)
$$

In the Thermodynamic limit $(N \rightarrow \infty ; j \rightarrow \infty)$, the effective hamiltonian can be derived by simply neglecting terms with $\mathrm{j}$ in the denominator, so it takes for the normal phase the following form:

$$
H=w_{0}\left(b^{+} b-j\right)+w a^{+} a+D\left(a+a^{+}\right)^{2}+\lambda\left(a+a^{+}\right)\left(b+b^{+}\right)
$$

By proceeding a simple diagonalization of this hamiltonian, one can find for the normal phase the following eigenvalues:

$$
E_{ \pm}=\sqrt{\frac{1}{2}\left(w^{2}+\frac{4 \lambda^{2} w_{0}}{w}+4 D w \pm \sqrt{\left(w^{2}+4 D-w_{0}^{2}\right)^{2}+16 \lambda^{2} w w_{0}}\right)}
$$

A similar approach can be done in the superradiant phase [22] by displacing the bosonic modes : $a^{+} \rightarrow c^{+}+\sqrt{\alpha}, \quad b^{+} \rightarrow d^{+}-\sqrt{\beta} \quad$, in order to capture the macroscopic behaviour of the system. These energies fully describe low-energy spectrum of the system. In the following, we plot the energy spectrum as a function of the light matter coupling strength for the case of the generalized Dicke model, the circuit QED and the cavity QED. 
We note first, that the variation of the energy spectrum as a function of the coupling constant $\lambda$ for the cavity QED, shows that the SPT is forbidden (see figure 1). This results show that the superradiant phase transition (SPT) is crucially affected by the value of the diamagnetic term D.

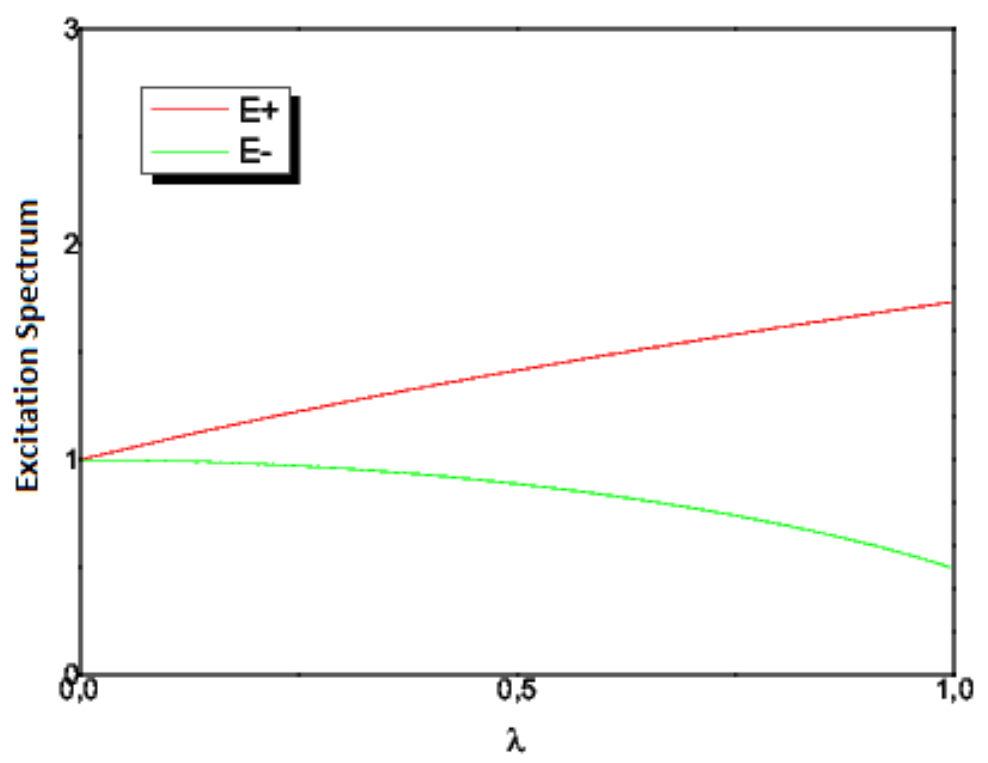

Figure 1. Energy spectrum as a function of the coupling constant $\lambda \square$ for the for the Cavity QED.

In circuit QED (figure 2) composed of a Cooper pair qubit in a transmission line resonator, which have a value of the minimal coupling included between $0<\mathrm{D} \prec \lambda^{2}$, a superradiant phase transition (SPT) for the Dicke model happens with a shift of the critical value.

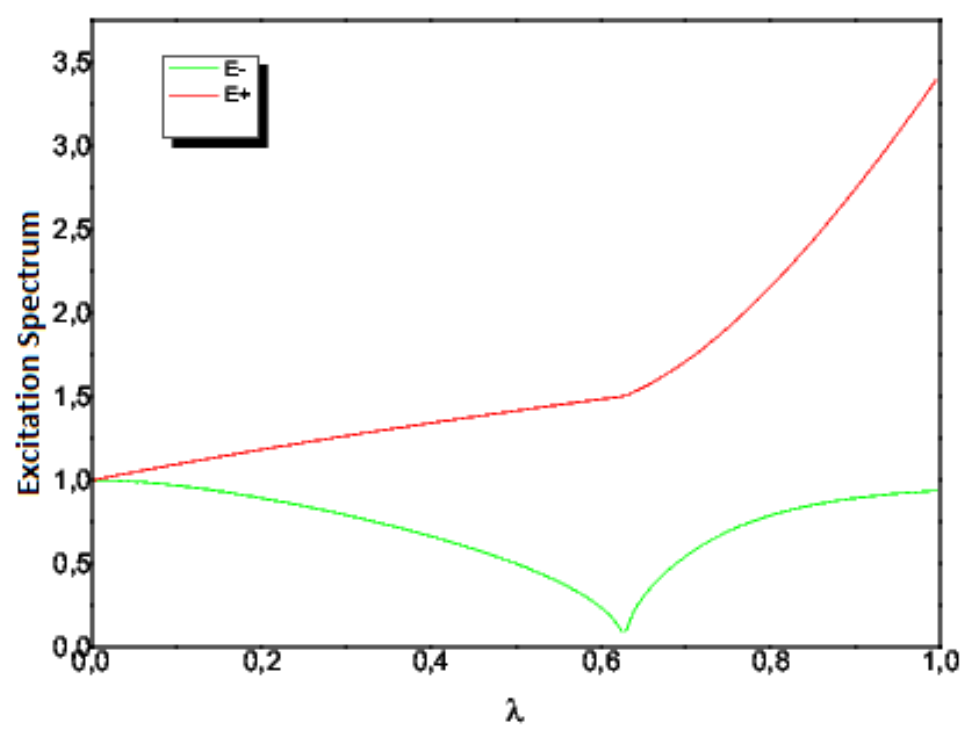

Figure 2. Energy spectrum as a function of the coupling constant $\lambda$ for the circuit QED.

In the next section, we proceed with a phase-space representation study. 


\section{Phase-space representation}

We have studied the energy spectrum of the Dicke model, that takes the form of two uncoupled harmonic oscillators and shown the existence of the superradiant phase transition in the system, allowing the identification of both quantum and semi-classical many-body features. This section intends to perform a more accurate study of the phase-space properties of the ground state wavefunction by paying a special attention to the diamagnetic term. The Dicke model ground state wavefunction can be obtained by the introduction of the position-momentum representation:

$$
\begin{aligned}
& x=\frac{1}{\sqrt{2 w}}\left(a+a^{+}\right) ; y=\frac{1}{\sqrt{2 w}}\left(b+b^{+}\right) \\
& p_{x}=i \sqrt{\frac{w}{2}}\left(a^{+}-a\right) ; p_{y}=i \sqrt{\frac{w_{0}}{2}}\left(b^{+}-b\right)
\end{aligned}
$$

The hamiltonian becomes

$$
H=\frac{1}{2}\left\{\left(w^{2}+2 D w\right) x^{2}+p_{x}^{2}+w_{0}^{2} y^{2}+p_{y}^{2}+4 \lambda \sqrt{w w_{0}} x y-w_{0}-w\right\}-j w_{0}
$$

and since the bi-partite system might be interpreted as a two-uncoupled harmonic oscillators, the ground state wave function might be written as,

$$
\Psi(x, y)=\left(\frac{E_{+} E_{-}}{\pi^{2}}\right)^{1 / 4} e^{-\left(E_{-} / 2\right)(c x-s y)^{2}-\left(E_{+} / 2\right)(s x-c y)^{2}}
$$

where $E_{ \pm}$is the excitation energies, $\tan (2 \gamma)=\frac{4 \lambda \sqrt{w w_{0}}}{w_{0}^{2}-\left(w^{2}+2 D w\right)^{2}}$ and $s=\sin \gamma ; c=\cos \gamma$ are the squeezing axis characterization parameters .

We will use in the following, the Wigner function for several reasons: firstly, it is suitable for analyzing the dynamics of a complex system closely to the classical limit, secondly, because it gives a rich framework for the representation of quantum dynamics using entirely the language of phase space variable and Finally, it is sensitive to the interference in phase space and consequently it provides a clear prediction to the possible occurrence of the nonclassical effects of the quantum mechanical system [23]. Recall that the Wigner function is given by:

$$
W(x, p)=\frac{1}{\pi \hbar} \int \Psi^{*}(x+y) \Psi(x-y) e^{2 i p_{y} / \hbar} d y
$$

where, $\mathrm{h}=1 \mathrm{x}, \mathrm{p}$ are the position and momentum parameters.

The Figures 3, 4, 5 represent a 2D numerical plot of the exact Wigner distribution function of the ground state as a function of the 'position' and the 'momentum'. Surprisingly, this function confirms the results previously found by the mean of the spectrum energy. For $0=\mathrm{D}$ and $0<\mathrm{D} \prec \lambda^{2}$, we observe that the Wigner distribution function in position and space is concentrated around $\mathrm{x}=\mathrm{p}=0$ for the normal phase, but for the superradiant phase a symmetry breaking is observed. The ground 
state wave function becomes doubly degenerate, it diverges and splits into two differentiated packets at the superradiant phase. This splitting can be regarded as a generation of a two schrodinger-cat like states. In contrast, for $\mathrm{D}=\lambda^{2}$, the Wigner function remains concentrated around $\mathrm{x}=\mathrm{p}=0$ and does not diverge at all.

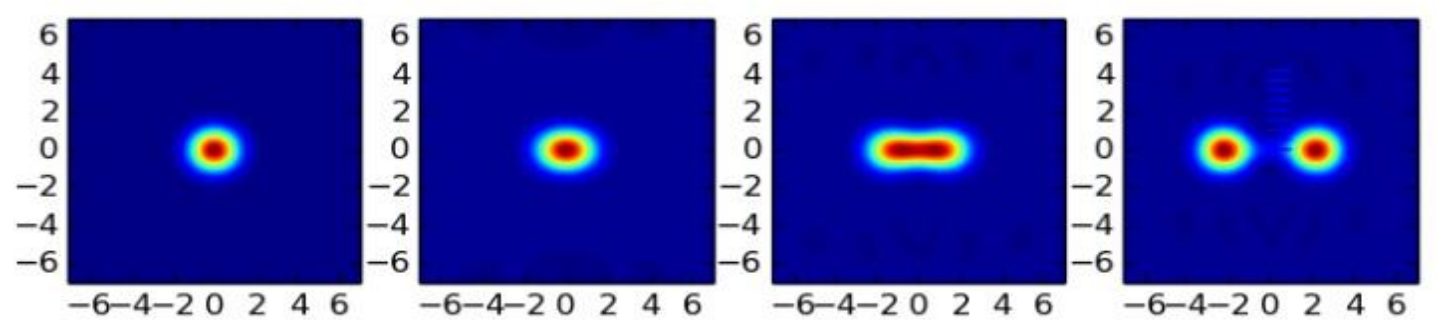

Figure 3. Phase Momentum presentation of the ground state wave function for the Generalized Dicke model for $($ lambda $=0.25,0.5,0.75,1)$
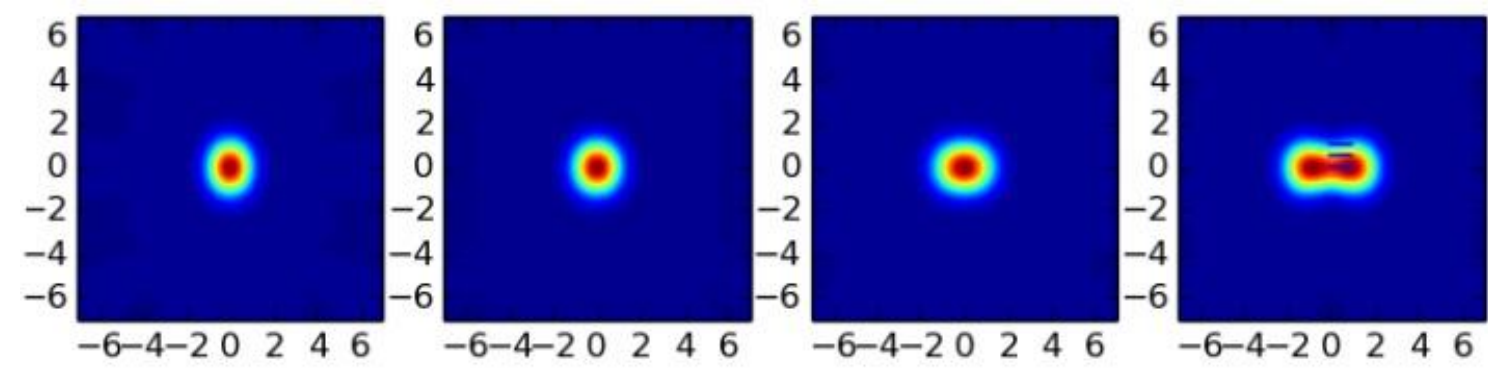

Figure 4. Phase Momentum presentation of the ground state wave function for the for the Circuit QED for $($ lambda $=0.25,0.5,0.75,1)$
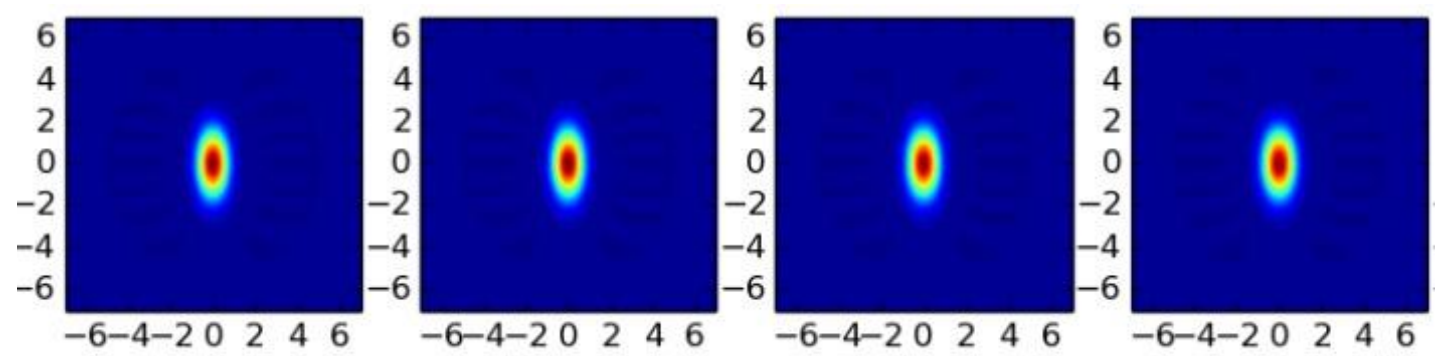

Figure 5. Phase Momentum presentation of the ground state wave function for the Cavity QED for $($ lambda $=0.25,0.5,0.75,1)$

\section{Entanglement}

The entanglement of the general Dicke model has been carried out by [24] and many others authors who study the entanglement for the $U(1)$ and the $Z_{2}$ symmetries $[25,26]$. But, to our knowledge, the influence of the diamagnetic term on the occurrence of the the entanglement have note been studied. The Dicke model exhibits a quantum phase transition at the thermodynamic limit, allowing the identification of both quantum and semi-classical many-body features. This quantum phase transition is well known as "The superradiant phase transition" and it represents a chaotic behavior and provides a promising way to well understand the entanglement, since large correlations and collective behaviour are an intrinsic part of critical systems. Entanglement is a striking feature of the quantum mechanics [27] and a key ingredient for quantum information processing. In effect, many concepts 
and formalisms can be used to quantify this one [28-30]. Here, we choose the Von Neumann entropy to measure the degree of entanglement for the bi-partite system:

$$
S\left(\rho_{R}\right)=-\operatorname{Tr}\left(\rho_{R} \log _{2} \rho_{R}\right)
$$

where $\rho_{\mathrm{R}}$ is the reduced density matrix.

The numerical results of the Von Neumann entropy (Figure 6) show that the squeezed states are a pure states. These latter become maximally entangled in the superradiant phase transition for both, the general Dicke model and the circuit QED ( $\mathrm{D}=0$ and $0 \prec D \prec \lambda^{2}$ ), hence the entanglement become less important and shifted wherever the D-term grows.

Moreover, the bi-partite system correlates without reaching the maximally entangled state for the case of the cavity QED $\left(\lambda^{2} \leq D\right)$. Thus, in accordance with the results found above in the phasespace representation section, one can conclude that the diamagnetic term plays a key role in limiting the maximally entangled states.

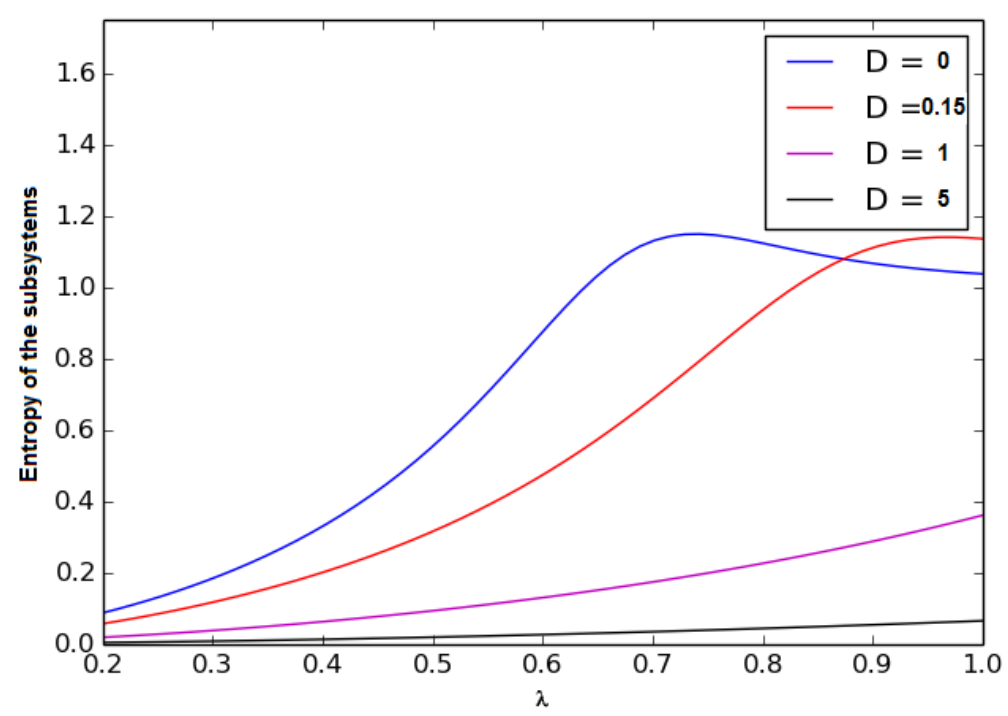

Figure 6. Von Neumann Entropy of the subsystems as a function of the interaction strength at resonance for the General Dicke model $(\mathrm{D}=0)$, the Circuit QED $(\mathrm{D}=0.15)$ and the Cavity QED $(\mathrm{D}=1$ and $\mathrm{D}=5)$

\section{Discussion and Conclusion}

We have studied the impact of the D term on the spectrum energy for several systems and shown that the subsystems start a second order phase transition beyond a critical value of the light-matter coupling if the counter rotating terms are present and the $\mathrm{D}$ term is negligible. We have also provided a numerical simulation of the Wigner distribution function underlying the important role of the D term on the the degeneration of the atomic states and in the limitation of the ground state wavefunction splitting. Finally, we have numerically shown the major impact of the diamagnetic term in the realization of a maximally entangled states.

We have also explored the ultrastrong coupling regime in the thermodynamic limit. We have firstly investigated the energy spectrum and shown the importance of the diamagnetic term. This latter affects the occurrence of the supperadiant phase transition and might prevent it. Furthermore, we 
have studied the Wigner distribution function and shown the quantum and semi-classical behaviour for different scenarios. Finally, we have outlined that the entanglement of the systems is limited by the presence of the diamagnetic term. Our theoretical work shows some interesting features of the cavity QED and the circuit QED in the thermodynamic limit introducing the diamagnetic term.

\section{References}

[1] Rabi, I. I. "Space quantization in a gyrating magnetic_eld." Physical Review, 51 (1937) 652.

[2] Dicke, R. H."Coherence in spontaneous radiation processes." Physical Review, 93 (1954) 99.

[3] Dimer, F., Estienne, B., Parkins, A. S., \& Carmichael, H. J. (2007). Proposed realization of the Dicke-model quantum phase transition in an optical cavity QED system. Physical Review A, 75(1), 013804.

[4] Mezzacapo, A., Las Heras, U., Pedernales, J. S., DiCarlo, L., Solano, E., \& Lamata, L.(2014). Digital quantum Rabi and Dicke models in superconducting circuits. Scientific reports, 4, 7482.

[5] Zhiqiang, Z., Lee, C. H., Kumar, R., Arnold, K. J., Masson, S. J., Parkins, A. S., \& Barrett, M. D. (2017). Nonequilibrium phase transition in a spin-1 Dicke model. Optica, 4(4), 424-429.

[6] M. H. Devoret and R. J. Schoelkopf, "Superconducting Circuits for Quantum Information: An Outlook." Science 339, 1169 (2013).

[7] Cirac, J. I., \& Zoller, P. (1995). "Quantum computations with cold trapped ions." Physical review letters, 74(20), 4091.

[8] Yoshihara, F., Fuse, T., Ashhab, S., Kakuyanagi, K., Saito, S., \& Semba, K. (2017). "Superconducting qubit-oscillator circuit beyond the ultrastrong-coupling regime." Nature Physics, 13(1), 44-47.

[9] Le Boité, A., Hwang, M. J., Nha, H., \& Plenio, M. B. (2016). "Fate of photon blockade in the deep strong-coupling regime." Physical Review A, 94(3), 033827.

[10] Aharonovich, I., \& Pe'er, A. (2016). "Coherent ampli_cation of ultrafast molecular dynamics in an optical oscillator." Physical review letters, 116(7), 073603.

[11] Carmichael, H. J., Gardiner, C. W., \& Walls, D. F. (1973). "Higher order corrections to the Dicke superradiant phase transition." Physics Letters A, 46(1), 47-48.

[12] Hepp, K., \& Lieb, E. H. (1973). "On the superradiant phase transition for molecules in a quantized radiation_eld: The Dicke Maser model." Annals of Physics, 76(2), 360-404.

[13] Nataf, P., \& Ciuti, C. (2010). "No-go theorem for superradiant quantum phase transitions in cavity QED and counter-example in circuit QED." Nature communications, 1, 72.

[14] Baden, M. P., Arnold, K. J., Grimsmo, A. L., Parkins, S., \& Barrett, M. D. (2014). "Realization of the Dicke model using cavity-assisted Raman transitions." Physical review letters, 113(2), 020408.

[15] Viehmann, O., von Delft, J., \& Marquardt, F. (2011). "Superradiant phase transitions and the standard description of circuit QED." Physical review letters, 107(11), 113602; Viehmann, O., von Delft, J., \& Marquardt, F. (2012). Reply to Comment on" Superradiant Phase Transitions and the Standard Description of Circuit QED". ; Ciuti, C., \& Nataf, P. (2012). "Comment on _Superradiant phase transitions and the standard description of circuit QED_. Physical review letters, 109(17), 179301.

[16] Nataf, P., \& Ciuti, C. (2010). "Is there a no-go theorem for superradiant quantum phase transitions in cavity and circuit QED?" Nat. Commun. 172

[17] Rzaewski, K., \& Wódkiewicz, K. (1991). "Stability of matter interacting with photons." Physical Review A, 43(1), 593. 
[18] Bamba, M., \& Ogawa, T. (2014). "Stability of polarizable materials against superradiant phase transition." Physical Review A, 90(6), 063825.

[19] De Liberato, S. (2014). "Light-matter decoupling in the deep strong coupling regime: The breakdown of the Purcell e_ect." Physical review letters, 112(1), 016401.

[20] Bialynicki-Birula, I., \& Rza»ewski, K. (1979). "No-go theorem concerning the superradiant phase transition in atomic systems." Physical Review A, 19(1), 301.

[21] Dicke, R. H. (1954). " Coherence in spontaneous radiation processes." Physical Review, 93(1), 99.

[22] Garbe, L., Egusquiza, I. L., Solano, E., Ciuti, C., Coudreau, T., Milman, P., \& Felicetti, S. (2017). "Superradiant phase transition in the ultrastrong-coupling regime of the two-photon Dicke model." Physical Review A, 95(5), 053854.

[23] Polkovnikov, A. (2010). "Phase space representation of quantum dynamics." Annals of Physics, 325(8), 1790-1852.

[24] Lambert, N., Emary, C., \& Brandes, T. (2004). "Entanglement and the phase transition in singlemode superradiance." Physical review letters, 92(7), 073602.

[25] Syljuåsen, O. F. (2003). "Entanglement and spontaneous symmetry breaking in quantum spin models." Physical Review A, 68(6), 060301.

[26] Yao, H., \& Qi, X. L. (2010). "Entanglement entropy and entanglement spectrum of the Kitaev model." Physical review letters, 105(8), 080501.

[27] Einstein, A., Podolsky, B. \& Rosen, N. "Can quantum mechanical description of physical reality be considered complete?" Phys. Rev 47, 777 (1935).

[28] Wootters, W. K. "Entanglement of formation of an arbitrary state of two qubits." Phys. Rev. Lett 80, 2245 (1998).

[29] Vidal, G. \& Werner, R. F. "Computable measure of entanglement." Phys. Rev. A 65, 032314 (2002).

[30] Vedral, V., Plenio, M. B., Jacobs, K. \& Knight, P. L. "Statistical inference, distinguishability of quantum states, and quantum entanglement." Phys. Rev. A 56, 4452 (1997). 